\title{
Global control of hepatitis C: where challenge meets opportunity
}

\section{David L Thomas}

\begin{abstract}
We are entering an important new chapter in the story of hepatitis C virus (HCV) infection. There are clear challenges and opportunities. On the one hand, new HCV infections are still occurring, and an estimated 185 million people are or have previously been infected worldwide. Most HCV-infected persons are unaware of their status yet are at risk for life-threatening diseases such as cirrhosis and hepatocellular carcinoma (HCC), whose incidences are predicted to rise in the coming decade. On the other hand, new HCV infections can be prevented, and those that have already occurred can be detected and treated-viral eradication is even possible. How the story ends will largely be determined by the extent to which these rapidly advancing opportunities overcome the growing challenges and by the vigor of the public health response.
\end{abstract}

\begin{abstract}
Most HCV morbidity and mortality occurs from chronic infection At the beginning of an $\mathrm{HCV}$ infection, hepatitis, jaundice and even fulminant hepatic failure can occur ${ }^{1}$. However, fulminant hepatic failure is rare, and most HCV infections are asymptomatic ${ }^{2-4}$. Cox et al. ${ }^{3}$ studied typical infections occurring in injection drug users in Baltimore. By monthly serologic testing before and during acute HCV infection, 62 persons had detectable acute $\mathrm{HCV}$ infection, but none of these individuals reported symptoms of sufficient severity to seek medical care ${ }^{3}$. In $10-40 \%$ of persons, the infection is self-limited; HCV-specific antibodies but not RNA are detected in blood, and there are no recognized long-term complications $s^{5}$.

In the majority of persons, $\mathrm{HCV}$ infection persists and can cause an array of outcomes, including cirrhosis and HCC. Cirrhosis can cause ascites, encephalopathy and/or variceal bleeding, a constellation of events referred to as end-stage liver disease or liver failure (Fig. 1). HCC can cause those same symptoms plus weight loss, jaundice, or fever and it typically occurs in those with cirrhosis 6 . Without treatment, cirrhosis and HCC can be fatal and explain most of the mortality directly attributed to $\mathrm{HCV}$ infection.

Cirrhosis, HCC or both occur over 30 years in a wide range (2-30\%) of all persons with chronic HCV infection ${ }^{7}$. The risks of cirrhosis and HCC increase with infection duration and are higher in persons who are concurrently infected with HIV, are infected at $>40$ years of age or consume more than the recommended amounts of alcohol ${ }^{8-10}$. In a study of 1,667 injection drug users positive for $\mathrm{HCV}$-specific antibodies and with an estimated median duration of infection of 14 years,
\end{abstract}

Division of Infectious Diseases, Johns Hopkins School of Medicine, Baltimore, Maryland, USA. Correspondence should be addressed to D.L.T. (dthomas@jhmi.edu).

Received 9 November 2012; accepted 5 April 2013; published online 8 July 2013; doi:10.1038/nm.3184 followed for over 12,737 person-years, only 40 had end-stage liver disease or HCC, an incidence of 3.1/1,000 person-years. However, the incidence was 3.6 times higher for those who consumed more than $260 \mathrm{~g}$ of alcohol per week and 3.7 times higher for those who were 38 years of age or older at enrollment compared to the reference groups ${ }^{9}$. On the other extreme, of 39 patients followed for an average of 9.7 years after acquiring $\mathrm{HCV}$ from blood transfusion during cardiac surgery, end-stage liver disease developed in $12 \%$ and cirrhosis in $20 \%$, among whom the mean age of infection was 58 years $^{11}$. Additional examples of even more rapid progression have been reported, often in persons infected at $\geq 60$ years of age or those with immunosuppression ${ }^{12,13}$. As the risks of cirrhosis and HCC increase progressively with age and duration of infection, future incidences are expected to rise (Box 1).

Extrahepatic diseases also cause morbidity. Persons with chronic HCV infection also have a high prevalence of multiple extrahepatic conditions compared to age-matched, general-population controls. For example, chronic HCV infection is strongly associated with mixed cryoglobulinemia vasculitis, a syndrome in which cryoglobulincontaining immune complexes deposit in small- and medium-sized blood vessels causing inflammation in skin, kidney and/or other tissues ${ }^{14}$. There are also complex associations between HCV and other conditions such as diabetes ${ }^{15}$, thyroid disease, lichen planus and an array of neuropsychiatric conditions such as depression (Fig. 1).

Collectively, the hepatic, extrahepatic and other related conditions are associated with a lower health-related quality of life for HCVinfected persons. In a systematic review, Spiegel et al. ${ }^{16}$ found that $\mathrm{HCV}$-infected persons scored lower than controls on all scales of the validated Short Form-36 quality-of-life survey. The largest difference in the HCV-infected group compared to controls was in the role-physical scale, where there was a mean difference of -15.8 points, followed by the role-emotional $(-13.0)$ and general health $(-12.6)$ scales. 
Figure 1 Outcomes associated with HCV infection. After acute infection, most persons develop viral persistence. Fatigue, joint pain, depression and a variety of skin manifestations can occur but are not usually diagnostic. In otherwise healthy persons infected at young ages, after decades some develop cirrhosis, end-state liver disease or HCC. There are wide ranges of the frequencies of these outcomes owing to differences in host factors such as race, age, HIV infection status and, for progression to end-stage disease, alcohol use.

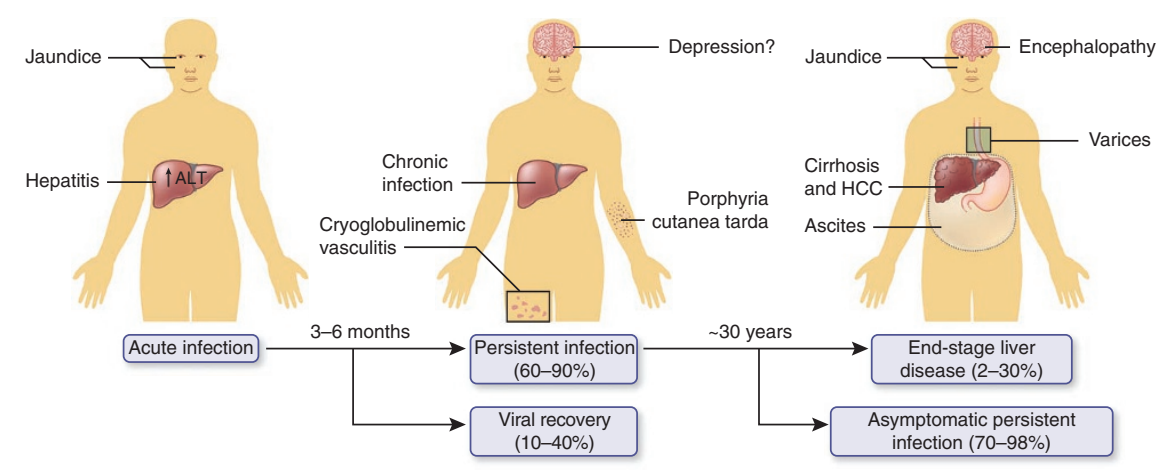

HCV transmission routes provide targets for prevention

Data on the global burden of disease reveal both the urgency of control measures as well as the strategies most likely to succeed. For example, the leading transmission routes are salient targets for preventive interventions (primary prevention), whereas data on the distribution and dynamics of existing infections are crucial to design strategies to manage disease (secondary prevention). These epidemiological data are presented first and then applied to specific control measures.

HCV can be transmitted by percutaneous inoculation of contaminated blood, through sexual intercourse (especially between men) and from a mother to her infant. In most instances the source of the virus is blood. Although HCV RNA has been amplified from semen, saliva, tears and urine, there is little evidence that these fluids are important sources of transmitted virus, possibly because there are too few intact viruses in these fluids and/or percutaneous exposure to them is uncommon 22 .

\section{BOX 1 Dynamic course of HCV infection}

In most regions of the world, the full impact of HCV infection is projected to rise as disproportionately affected age cohorts cross thresholds of infection duration or age that increase disease risk. The most detailed modeling has occurred for the United States 20,56 _ at least two-thirds of persons with HCV infection in the United States were born between 1945 and 1965 and probably acquired HCV infection $25-45$ years ago 51 . The first national survey was done near 1990, and the peak age of infected persons was 30-39 years (Fig. 3). When the survey was repeated 10 years later on another randomly selected segment of the population, the mean age of HCV-infected persons was then 40-49 years. Assuming that cirrhosis and HCC incidences start to climb after 30 years of infection and as persons reach more than 60 years of age, it is anticipated that the prevalence of both forms of disease will sharply increase in the next decade.

Davis and co-workers ${ }^{56}$ built a model that predicted 29,090 HCV liver-related deaths from 1980 to $1989,56,377$ from 1990

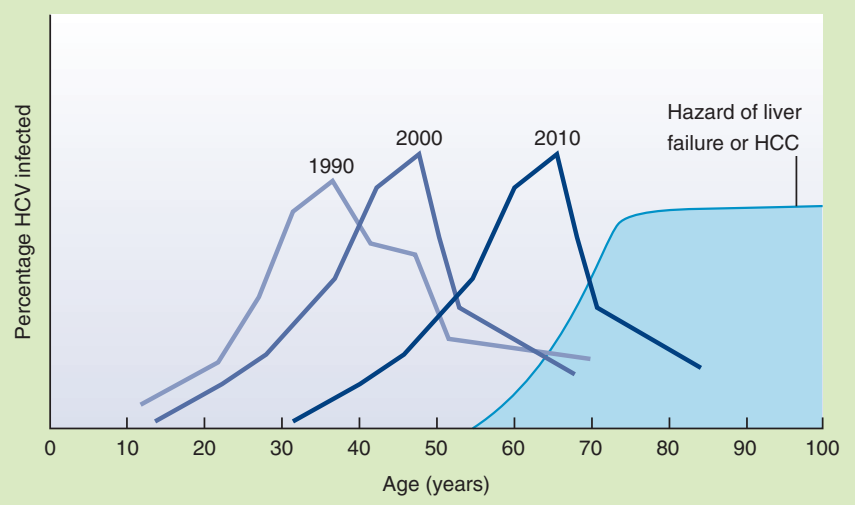

Figure 3 Dynamic course of HCV infection in the US. As the cohort born between 1945 and 1965 ages, a greater fraction will have HCV infection for sufficient time at old-enough ages to develop liver failure and HCC. to $1999,145,667$ from 2000 to $2009,254,550$ from 2010 to 2019 , and a peak at 283,378 from 2020 to 2029 . As the model accurately predicted what was actually observed before 2009 , the projections seem reliable. Another group predicted the same trend but with a later peak and lower rates: 38,600 new cases of end-stage liver disease, 3,200 referrals for transplant and 36,100 deaths between the years 2030 and 2035 (ref. 96). Others have added commensurate projections for the escalating economic impact of HCV infection ${ }^{97}$.

Likewise, in Egypt, HCV infection is projected to produce 127,821 deaths from cirrhosis and 117,556 deaths from HCC from 2009 to 2028 , prematurely ending 32.86 million years of life compared to a noninfected cohort ${ }^{98}$. In France, HCV-related HCC mortality is projected to increase through 2020 (ref. 99). With few exceptions ${ }^{100}$, most countries have yet to experience the peak burden of HCV infection. Fortunately, these projections do not account for improved efforts to control morbidity and mortality by prevention and treatment. 
The size of the inoculum matters. HCV infection is nearly universal when a unit of contaminated blood is transfused but occurs in only 1 to 5 per 100 single needle-stick exposures and almost never when HCV RNA cannot be recovered from donor blood that contains HCV-specific antibodies ${ }^{23-25}$. There is also a correlation between the concentration of HCV in the exposed blood and the likelihood of transmission. For example, HCV is more likely to be transmitted from a mother to her infant when the mother is also HIV infected and has higher HCV RNA levels ${ }^{26-28}$. The virus also seems to be relatively stable in syringes, on gauze or in water bottles ${ }^{29,30}$. In one study, a high $\mathrm{HCV}$ inoculum remained infectious in cell culture after the virus had been stored in bottled water for 3 weeks ${ }^{30}$.

Although percutaneous exposures cause most HCV infections, permucosal infection also occurs. Permucosal transmission has been reported when HCV-contaminated blood is splashed into the eyes ${ }^{31}$, but it more often refers to transmission by sexual intercourse or during intranasal cocaine use ${ }^{32,33}$. There is some controversy regarding the efficiency of sexual transmission. On the one hand, HCV infection is associated with high-risk sexual exposures, and there are outbreaks reported among men who report no other risk than having sex with other men ${ }^{32,34,35}$. On the other hand, HCV transmission is rare among long-term heterosexual partners of $\mathrm{HCV}$-infected persons ${ }^{36,37}$. These disparate findings could be reconciled by the enhancement of transmission by permucosal tears, which are more common in men who have intercourse with other men than in people in long-term monogamous heterosexual relationships. HCV also could be more transmissible in the acute phase of infection, which might lead to sexual spread early after infection occurs but which might be missed in studies of monogamous, long-term relationships.

Worldwide, the frequencies of HCV transmission by various routes differ, and major changes in frequencies have occurred in some regions of the world ${ }^{38}$. In economically developed regions, injection drug use is the dominant transmission route. For example, in the last decade in the United States, injection drug use accounted for more than two-thirds of HCV infections ${ }^{39}$. Moreover, the incidence of HCV infection is 5-30 per 100 person-years in illicit injection drug users ${ }^{3,40}$. Transfusions were once a major form of HCV transmission and accounted for almost one-quarter of cases. However, transfusion transmission of HCV has been nearly eliminated in regions of the world where donations are tested for HCV-specific antibodies and HCV RNA ${ }^{41}$.

HCV transmission has also been clearly demonstrated from percutaneous exposures in the context of traditional and some nontraditional medical care. As with transmission by transfusion, transmission of $\mathrm{HCV}$ in medical practice has declined in many regions, probably because of expanded recognition of blood-borne pathogens and adoption of universal precautions. Where such measures are observed, exposure to medical care is not reported statistically more often by persons with acute HCV infection compared to controls. However, sporadic transmission is still well documented when there are breaches in optimal infection control practices ${ }^{42,43}$. For example, between 1998 and 2005 , recent exposure to a medical practice was the only exposure reported by $67 \%$ of 109 persons in Spain with new HCV infection $^{43}$. Moreover, in economically developing regions of the world, it is likely that this form of HCV transmission is the predominant route of spread. The World Health Organization (WHO) has estimated that each year in economically developing regions there are 3.4 injections per person, 39\% performed with reused medical equipment, causing 2 million new HCV infections ${ }^{44}$.

Likewise, other percutaneous practices such as tattooing, scarification rituals, circumcisions and acupuncture have transmitted HCV infections in the past, and they continue to be important modes of transmission when proper sterilization techniques are not observed ${ }^{45}$. As discussed below, one challenge is acquiring the resources and education needed to eliminate these unsafe procedures.

\section{Infection prevalence is global but uneven}

A major challenge to control of $\mathrm{HCV}$ infection is the large number of persons already infected and their location in some of the poorest regions of the world. In many cases, estimates are based on very limited data. The WHO recently revised its estimates such that in 2005 there were more than 185 million HCV-specific antibodypositive persons, or $2.8 \%$ of the human population, up from 122 million and $2.3 \%$ in 1990 (ref. 46); 130-170 million are believed to be chronically infected. However, there are marked differences in HCV prevalence between regions (Fig. 2) and between age and risk groups within regions. Egypt seems to have the highest national $\mathrm{HCV}$ prevalence, up to $50 \%$ in persons born before 1960 (ref. 47), and the history of HCV infection in Egypt is exemplary of global transmission patterns. From the 1950s to the 1980s, there was a campaign in Egypt to eradicate schistosomiasis infection by intravenously administering tartar emetic to millions of residents ${ }^{48}$. Without proper sterilization procedures, HCV was transmitted extensively, and HCV prevalence is $15-50 \%$ among persons who were alive during that campaign, whereas it is $1-2 \%$ in those born later. Similar age cohorts have been described elsewhere in the world and have been linked to unsafe infections or percutaneous practices that no longer occur ${ }^{49,50}$.

The best data on HCV prevalence in the Unites States come from the serial National Health and Nutrition Examination Surveys $(N H A N E S)^{51}$. By testing blood collected from a subset of persons representing households in the United States from 1988 to 1994, it was estimated that 3.9 million individuals, or $1.8 \%$ of the general population, were infected with HCV. Omitted from this survey were nearly 2 million incarcerated persons in the United States, among whom there are probably another 500,000 infected with $\mathrm{HCV}^{52}$. The survey was repeated a decade later in a new sample of the population, and the same age cohort of infected individuals was evident, now 10 years older (Box 1, Fig. 3) ${ }^{51}$. On the basis of these data, it is estimated that more than three-quarters of all HCV-infected persons in the United States were born between 1945 and 1965, an epidemiological observation that has had a direct impact on strategies for control (discussed below).

Infection prevalence is higher among racial minorities than in Americans of European descent. In a 2000 NHANES report, in nonHispanic blacks $40-49$ years of age, the HCV prevalence was $14 \%$ compared to a general population prevalence of $1.6 \%$. HCV infection is also strongly linked to injection drug use, and $50-80 \%$ of injection drug users are infected with $\mathrm{HCV}^{39,53}$. Thus, just as injections for schistosomiasis in the 1950s-1980s yielded a cohort of HCV-infected persons in Egypt, in approximately the same period in the US, expansion of illicit drug use, unsafe medical practices and contaminated transfusions created the 1945-1965 age cohort of HCV infection.

\section{Cirrhosis and HCC prevalence}

There are no reliable estimates of the number of persons living with HCV-related cirrhosis or HCC, and even mortality figures are imprecise. The WHO estimated that in 2002 there were 783,000 deaths from cirrhosis, and $27 \%$ of all cases were attributed to HCV infection $^{54}$. The highest percentage of cirrhosis attributed to HCV (62\%) was reported from a Western Pacific region that included Japan and Australia, whereas the lowest percentage (16\%) derived from 


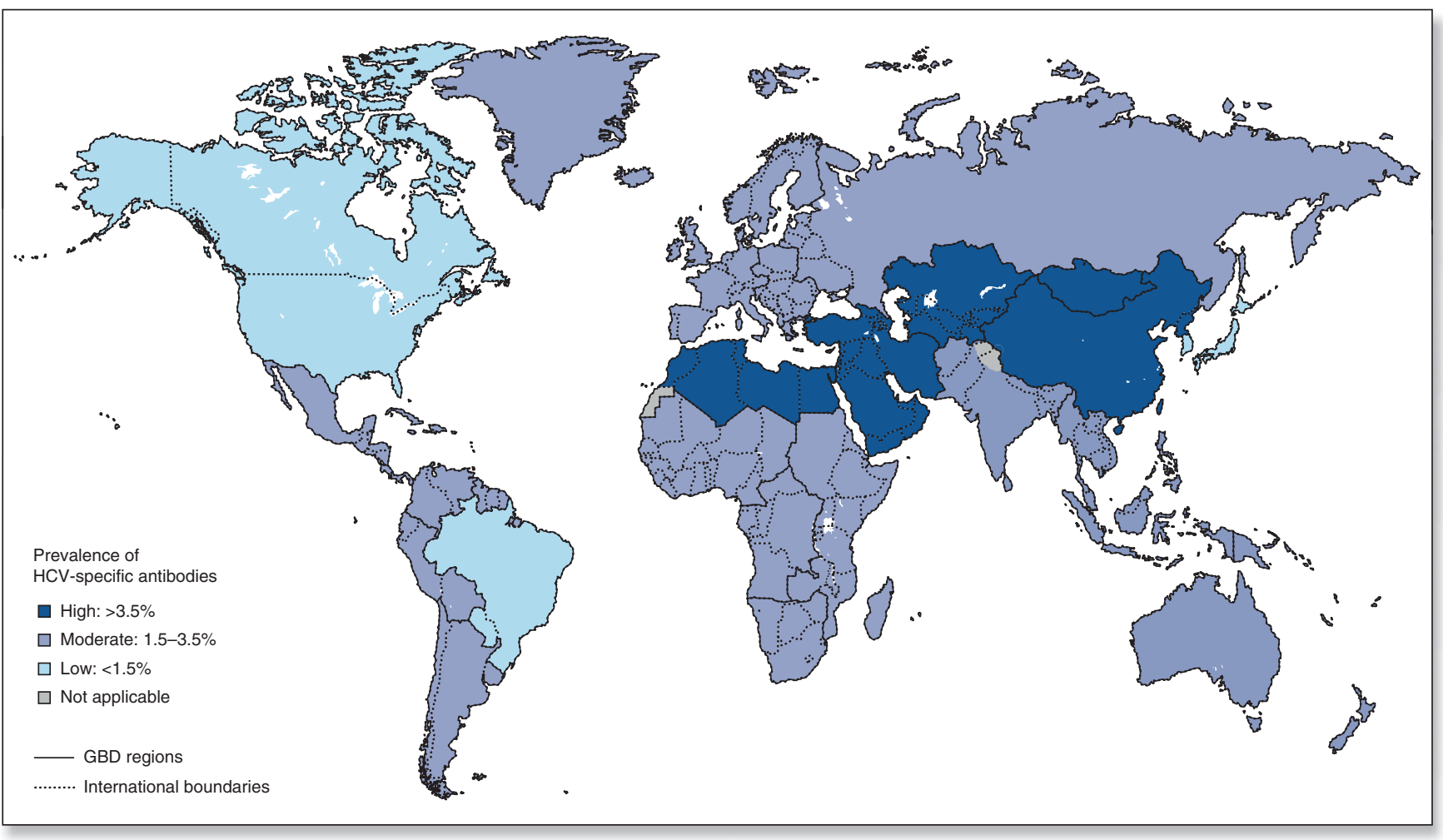

Figure 2 Global prevalence of HCV-specific antibodies. On the basis of a meta-analysis of 232 studies published in 1997-2007, point prevalences are calculated using regional population weights ${ }^{46}$. GBD, global burden of diseases.

sub-Saharan Africa. In another comprehensive review of global mortality, deaths from HCV-related cirrhosis were estimated at 287,400 in 2010 , a $35.6 \%$ increase from the rate in 1990 (ref. 55). The prevalence of HCV-related cirrhosis has also been modeled using estimates of the number of infected persons and distribution of disease cofactors. For example, Davis and co-workers constructed various models of cirrhosis in the United States on the basis of an assumption of 3.49 million persons infected in 1994 and published estimates of the time to develop cirrhosis ${ }^{56}$. In 2006 they estimated $20 \%$ of those with persistent HCV infection had cirrhosis, or approximately 650,000 persons; the number with cirrhosis in the United States is expected to rise (Box 1, Fig. 3).

Data on HCC are better than on cirrhosis because most primary liver cancer is due to HCC, and there is a high fatality rate. In 2002, the WHO estimated that there were 619,000 deaths from HCC and attributed $25 \%$ of those to $\mathrm{HCV}$ infection ${ }^{54}$. In another comprehensive review of global mortality, deaths from HCV-related HCC were estimated at 195,700 in 2010, a 73.3\% increase from the number in 1990 (ref. 55). Marked global differences in HCV-related HCC incidence and the proportion of HCC due to HCV infection are explained by differences in HCV and hepatitis B virus (HBV) prevalence and the ages of most persons with the two chronic viral infections. For example, HCV is estimated to cause just $\sim 15 \%$ of HCC in Taiwan, and HBV is the dominant cause of HCC ${ }^{57}$. In contrast, in Japan HCV causes most HCC, and HCV-infected persons in Japan have the highest rates of $\mathrm{HCC}^{58}$. Although genetic and environmental differences between countries may contribute to these epidemiological differences, there is increasing evidence that the older age of HCV-infected persons in Japan (relative to other countries) is largely responsible for the tight HCV-HCC association in Japan, whereas in Taiwan the once widespread transmission of $\mathrm{HBV}$ to infants seems to explain the current dominance of $\mathrm{HBV}$ as a cause of $\mathrm{HCC}^{59}$. If so, this observation would suggest that a similar epidemic of HCV-related HCC will occur in Europe and the United States when the average age of infected persons approaches that in Japan.

\section{Hepatitis $\mathrm{C}$ can be controlled}

The two major approaches to controlling HCV infection are prevention of new infections (primary prevention) and management of existing infections (secondary prevention) (Table 1). The strategies differ, but the results are complementary. As treatment eliminates infection and humans are the only source of virus, treatment can also be a form of prevention. Likewise, as re-infection negates the benefits of treatment, prevention can be a form of treatment.

Infection can be prevented. As with any infectious disease, the optimal method of HCV control is prevention. HCV transmission by transfusion has been virtually eliminated by testing of donations for $\mathrm{HCV}$-specific antibodies and HCV nucleic acid ${ }^{60}$. Likewise, implementation of universal precautions and standard infection control procedures prevents HCV transmission by medical practices. There is also evidence that the incidence of HCV infection is declining among injection drug users. For example, Mehta et al. ${ }^{61}$ reported that the HCV infection incidence declined in consecutive injection drugusing cohorts in Baltimore from 1988 through 2008.

$\mathrm{HCV}$ treatment efficacy is rapidly improving. The success of $\mathrm{HCV}$ treatment is chiefly measured by the proportion of persons in whom HCV RNA can no longer be detected in blood by the end of treatment and 6 months after treatment is stopped, called a sustained virologic 


\section{Table 1 Key elements in HCV control program}

Prevent infection (primary prevention)

Test blood supply for HCV-specific antibodies and HCV RNA

Counsel infected persons to eliminate transmission-prone practices

Observe universal precautions in traditional and nontraditional medical practices

Reduce illicit drug use and improve safety

Investigate and implement vaccination

Prevent disease (secondary prevention)

Test to detect infection

Vaccinate for hepatitis A virus and hepatitis $B$ virus

Reduce or eliminate alcohol use

Treat with antivirals

response (SVR). Because relapse of infection is rare more than a few months after treatment is stopped, SVR is often used interchangeably with cure. Since interferon- $\alpha$ was first approved by the US Food and Drug Administration for treatment of HCV infection in 1991, all licensed therapies have included some form of interferon- $\alpha$ injection. Responses were improved in 1998 by combining interferon- $\alpha$ with oral ribavirin and then in 2001-2002 by linking the interferon molecule with polyethylene glycol, producing peginterferon- $\alpha^{62-64}$. For infection with genotype $1 \mathrm{HCV}$, an additional advance came in 2011 with the approval of the HCV protease inhibitors telaprevir and boceprevir ${ }^{65,66}$. Accordingly, there has been progressive improvement in HCV treatment efficacy. The SVR rate for the most difficult-to-treat genotype $1 \mathrm{HCV}$ infection was $\sim 40 \%$ in 2010 (ref. 67 ) and $\sim 66 \%$ in 2011 (refs. 65,66) and is anticipated to be $>75 \%$ by 2014. (Actual SVR figures depend on multiple factors including the percentage of patients who are black or have cirrhosis.)

There is clear evidence that much of the morbidity and mortality associated with HCV infection can be controlled by treatment. Successful treatment of HCV infection is associated with a reduced incidence of liver disease progression, including cirrhosis, end-stage liver disease and $\mathrm{HCC}^{68-71}$. A study of persons with advanced fibrosis or cirrhosis (Ishak fibrosis stage 4-6) who received interferon-based HCV treatment between 1990 and 2003 followed 530 patients over a median of 8.4 years ${ }^{72}$. The ten-year mortality rate among persons with SVR was $8.9 \%$, compared to $26.0 \%$ among those without SVR. The ten-year liver-related mortality/transplant rate was $1.9 \%$ with SVR compared to $27.4 \%$ without SVR. Another study of US veterans who started HCV treatment between January 2001 and June 2007 found a clear survival benefit to those who achieved SVR ${ }^{73}$. Among more than 17,000 persons, SVR was associated with a reduced hazard of death of 0.51 to 0.70 , depending on the HCV genotype. Whereas these findings may reflect greater treatment response in persons with less pretreatment disease (rather than a treatment effect), adjustments were made for pretreatment fibrosis stage, and placebo-controlled studies are not ethical. Moreover, a recent systematic review found clear evidence that successful therapy reduced the incidences of liver failure, HCC and mortality ${ }^{74}$.

Similarly, successful treatment of $\mathrm{HCV}$ infection can improve quality of life $\mathrm{e}^{75,76}$. Bernstein et al. ${ }^{75}$ evaluated the change in healthrelated quality-of-life reports from baseline to end of follow-up for 1,441 persons from international studies of HCV treatment. Improvements in quality of life were strongly associated with successful treatment. Likewise, another study reported better quality-of-life results, fewer absences from work and greater involvement in volunteer and household activities among HCV-infected persons with successful treatment responses compared to those whose treatment was unsuccessful $^{76}$. Thus, successful treatment has the capacity to reduce many of the complications of HCV infection.

\section{Challenges to control by prevention}

Although there is evidence that HCV infection can be prevented, there are abundant examples that all prevention efforts need to be intensified. Vaccination is a major tool for prevention of many infectious diseases. However, HCV is genetically and antigenically diverse, and the immunologic correlates of protective immunity are only incompletely understood. These, and other factors, have constrained HCV vaccine development (see Review in this issue of Nature Medicine by Liang ${ }^{77}$ ). Thus, HCV infection is prevented mainly by eliminating exposures or making them safer.

Although we know how to prevent HCV transmission from healthcare practices, the leading route of $\mathrm{HCV}$ transmission worldwide is unsafe medical procedures $44,46,47,49,50,78$. As mentioned above, the WHO estimates there are 2 million new HCV infections each year as a result of unsafe infection ${ }^{44}$. Even in Europe and the United States, $\mathrm{HCV}$ transmission during routine health procedures continues when there are breaks in infection control practices ${ }^{43,78}$. Clearly, education about avoiding unnecessary injections is key, as is information on universal precautions and resources to implement them.

The second leading global route of HCV transmission is probably illicit drug use. Although in some settings HCV incidence has declined among injection drug users, new infections still occur at incidences of $5-30 \%$ per year ${ }^{61,79}$. Measures such as needle exchanges may have contributed to the reduced incidence in some places ${ }^{80}$ but have not been sufficient and, from a global perspective, have had a minimal impact on HCV transmission. Data demonstrating the stability of virus in syringes and drug-use equipment need to be translated into practices that eliminate transmission risk when drug use itself cannot be stopped.

Likewise, there are major challenges in preventing HCV transmission in other contexts. Multiple outbreaks of new HCV infections have been reported among male homosexual populations ${ }^{81}$, and, as with the case in injection drug users, in some instances these infections have negated the benefits of prior successful treatment ${ }^{82}$. Methods to prevent transmission from a mother to her infant (such as cesarean section, maternal treatment and infant vaccination) that have worked for other infectious diseases have either not worked for $\mathrm{HCV}$ or are not yet possible ${ }^{83}$. In this regard, development of HCV treatment that is not toxic to the fetus is particularly promising but not currently available. Thus, the available evidence demonstrates that infection can be (and is) prevented but that current control efforts have to be intensified in these key settings.

\section{Challenges to control by treatment}

Lack of recognition of infection is a major impediment to controlling existing infection. In the United States, it is estimated that less than half of HCV-infected individuals are aware of their infection. In a 2001-2008 NHANES survey of US households, 393 HCV-specific antibody-positive persons were identified, and 170 were contacted after being notified of their HCV status through the study ${ }^{84}$. Only $49.7 \%$ were already aware of their HCV infection before being notified by the study personnel. Interestingly, only $3.7 \%$ had been tested because their doctor followed the 'risk-based' testing strategy in effect since 1998. Given that this study excluded inmates, the homeless and other populations at highest risk of unrecognized HCV infection, the true prevalence of unrecognized infection may be more than $60 \%$. Even treatments of $100 \%$ efficacy will have no impact on unrecognized infection (Fig. 4). 


\section{BOX 2 Breaking tolerance is critical to control of HCV infection}

There is an interesting parallel between the manner in which HCV has evolved mechanisms to replicate in hepatocytes and the persistence of HCV infection in society. At the cellular level, Gale, Lemon and others have characterized the elegant mechanisms through which the HCV protease silences the cellular sensors of RNA replication such as the retinoic acid inducible gene I (ref. 101). Although these and other molecules recognize pathogen-associated molecular patterns on HCV RNA, the virus dampens downstream signaling cascades and net immune responses sufficiently to persist in most persons.

Likewise, HCV replication is not commonly recognized by the host. Acute infection rarely causes sufficient symptoms to lead an infected person to seek medical care. Clinically silent acute infections are more likely to cause long-term complications than symptomatic ones, both because they are more likely to persist and because persons with unrecognized infections cannot be treated or cautioned to prevent further transmission2,102. During the vast majority of HCV's subsequent natural history, replication continues without production of sufficient symptoms to provoke a person to seek medical help. The virus also disproportionately affects segments of society less apt to receive medical treatments, further enhancing its odds of persisting.

Control of infection both within a cell and within society requires that this tolerance for HCV replication be broken. Medications that penetrate into hepatocytes and stop replication within that cellular compartment have been developed. Although these medications are clearly able to eradicate infection, they have not yet penetrated into most segments of society where replication continues unchecked. From the virus's perspective, prisoners, illicit drug users and all individuals with unrecognized HCV infection represent protected replication sanctuaries.

All projections call for a sharp rise in the incidence of HCV-related cirrhosis and HCC. It is necessary for the danger of this threat to signal society to expand HCV testing and treatment access to prevent these outcomes. The degree of success is likely to be commensurate to the vigor with which the silence is overcome.

Rates of treatment uptake among those with recognized infection are also low. In Europe, by 2006 it was estimated that chronic hepatitis $\mathrm{C}$ treatment was provided for 308,000 persons who comprised no more than $16 \%$ of the $\mathrm{HCV}$-infected persons in any individual country ${ }^{85}$. In the United States, from 2002 to 2007 an estimated 663,000 of the $\sim 4$ million HCV-infected persons were treated ${ }^{86}$. In one analysis of nearly 100,000 US veterans with chronic hepatitis C, only $11.6 \%$ received and $6.4 \%$ completed treatment ${ }^{87}$.

Effective HCV control measures exceed the available resources in many regions of the world and segments of society where infection burden is greatest. In the United States, the average wholesale cost of the medications for one course of current HCV treatments for genotype $1 \mathrm{HCV}$ infection can exceed $\$ 100,000$. This is certainly an extreme example, and in other regions of the world generic drugs can be obtained for a fraction of that price. Nonetheless, peginterferon- $\alpha$ is currently necessary for all forms of HCV treatment but by early 2013 was not on the WHO List of Essential Medications. In addition,

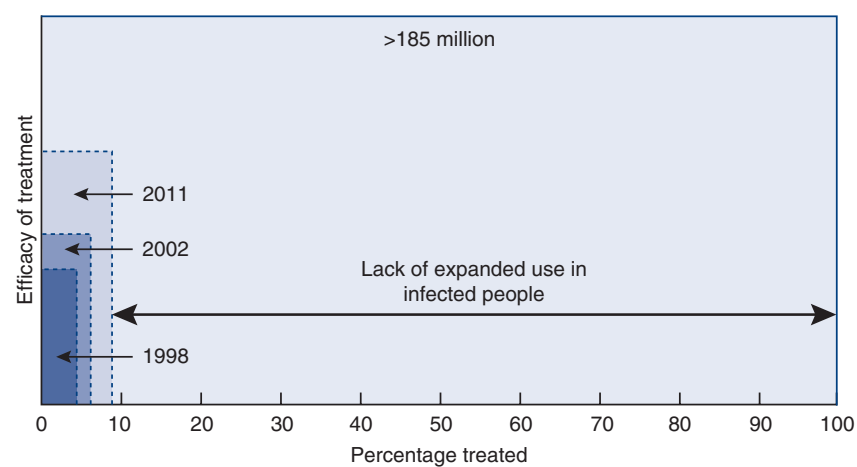

Figure 4 Importance of expanding testing and treatment to impact the global prevalence of HCV infection. Because the percentage cured is a function of the distribution of many variables such as genotype, age, and race, the $y$ axis arbitrarily shows the efficacy of treatment, which is increasing but having little impact on the overall burden of disease represented by the total area of the figure. In 2005, an estimated 130-170 million individuals had chronic HCV infection, whereas >185 million individuals had HCV-specific antibodies (ref. 46). beyond the drug costs, there are other issues such as the availability of laboratory testing, refrigeration of drugs, and monitoring of treatments that contribute additional costs and complexities that make it extremely challenging to initiate widespread treatment of $\mathrm{HCV}$ infection in some regions of the world.

\section{HCV control challenges can be overcome}

No more than $15 \%$ of the $>185$ million HCV-specific antibody-positive persons worldwide are aware of their status, despite the availability for more than 20 years of an accurate screening test. Thus, one of the most important elements in a successful control program has to be expanded HCV testing. Methods are now available for rapid testing for HCV-specific antibodies at the point of care ${ }^{88}$. Given that in some societies there is stigma around HCV infection, anonymous testing options also should be made available. With all screening, it is important to add HCV RNA testing for persons in whom HCV-specific antibodies are detected by screening. Unfortunately, RNA testing requires a specialized laboratory.

In August of 2012, the US Centers for Disease Control and Prevention recommended a new HCV screening strategy focused on all individuals born between 1945 and 1965, the cohort with the highest HCV prevalence ${ }^{89}$. As mentioned above, this cohort is believed to comprise at least two-thirds of all HCV-infected persons in the United States. In a recent analysis of the cost-effectiveness of this approach, the Centers for Disease Control and Prevention estimated that 1.9 million persons from this age group had chronic HCV infection in 2006 and saw a healthcare provider at least once ${ }^{20}$. Their models suggest birth cohort testing would identify $1,070,840$ new cases of chronic hepatitis $\mathrm{C}$, a measure that would reduce the morbidity and mortality predicted in this cohort in the next 10-20 years. Given current treatment efficacy, full implementation of this testing recommendation might prevent 121,000 deaths and be cost effective compared to most accepted practices such as colorectal cancer screening. Similar methods should be employed elsewhere, even before the widespread availability of treatments, since those who are infected can benefit from counseling to delay progression of disease by limitation of alcohol ingestion, to be vaccinated against hepatitis A virus and $\mathrm{HBV}$, and to prevent secondary transmission. 
Lok et al. ${ }^{90}$ have published proof that interferon-free treatments are possible, and even more encouraging results have already been reported for phase 2 studies. For example, Poordad et al. ${ }^{91}$ reported that $93-95 \%$ of $33 \mathrm{HCV}$ genotype 1-infected persons achieved SVR 12 weeks after stopping treatment with various doses of an inhibitor of the HCV NS3 serine protease (ABT-450 coformulated with ritonavir, $\mathrm{ABT}-450 / \mathrm{r}$ ), along with a non-nucleoside inhibitor of the NS5b HCV polymerase (ABT-333) and ribavirin. ABT-450/r has since been coformulated with an inhibitor of HCV NS5A (ABT-267) and moved into phase 3 testing to examine the safety and efficacy with ABT-333 and ribavirin. Likewise, Gane et al. ${ }^{92}$ reported that 21 of 25 HCV genotype 1-infected persons and 10 of $10 \mathrm{HCV}$ genotype 2- or HCV genotype 3-infected persons achieved SVR when treated with a nucleotide inhibitor of the HCV polymerase (sofosbuvir) along with oral ribavirin for 12 weeks. In another study, sofosbuvir was combined with an inhibitor of NS5A (GS5885) and ribavirin, and produced SVR at least 4 weeks after treatment was stopped in 25 of $25 \mathrm{HCV}$ genotype 1-infected persons ${ }^{93}$. The compounds sofosbuvir and GS5885 were coformulated into a once-daily, fixed-dose medication that has moved into phase 3 testing of one pill once a day for treatment of genotype $1 \mathrm{HCV}$ infection with or without ribavirin. Also noteworthy are the adverse-event profiles of these medications, which seem much safer than the currently approved peginterferon-based treatments. Thus, oral medications that can cure HCV more often, in less time and with fewer side effects are coming rapidly.

Although interferon-sparing HCV treatments are not yet approved for clinical use, there are obvious ways in which they will improve HCV control. Persons who are intolerant to interferon or for whom interferon-based therapies are not efficacious will now have additional treatment options. In addition, it is possible that a growing perception of greater treatment safety and efficacy will encourage more patients to opt for therapy. Simpler, safer and more efficacious treatments might also make primary-care providers expand testing, a crucial need, given that $<50 \%$ of infected individuals are aware of their status. As HCV is not transmissible after SVR, more effective HCV treatment should also reduce new infections. Prevention of transmission by treatment has worked to varying extents for other infections such as HIV, tuberculosis and syphilis. However, the degree to which $\mathrm{HCV}$ treatment will reduce transmission within populations or in particular settings such as from a mother to her infant remains to be demonstrated.

Hepatitis $\mathbf{C}$ eradication is the goal. The example of smallpox makes it clear that eradication is the most successful method of controlling infectious diseases. Notably, HCV infection has many attributes that make an infection a candidate for eradication (Table 2). Blood-borne infections are much easier to control than those transmitted by aerosols or food. In addition, existing therapies can permanently eliminate $\mathrm{HCV}$ infection. Because there is no natural nonhuman HCV reservoir, elimination of infection from individuals effectively prevents subsequent transmission. Thus, even though many infected populations such as injection drug users do not actively seek medical care, treatment efforts need to be sustained for only finite periods of time to cure a given host. Treatment of enough individuals could eliminate transmission once the $\mathrm{HCV}$ reservoir drops below what is necessary to sustain the epidemic, even if risk practices themselves cannot be controlled.

On 21 May 2010, the World Health Assembly passed a resolution that called for the WHO to develop a comprehensive approach to control chronic hepatitis. In July of 2012, the WHO announced a framework for global action for the prevention and control of
Table 2 Attributes of an infectious disease that can be eradicated

\begin{tabular}{ll}
\hline Attribute & Relevance to HCV \\
\hline Can be cleared from host & True \\
Transmission can be controlled & True \\
No natural nonhuman reservoir & True \\
Adequate public health infrastructure & $?$ \\
Sufficient funding & $?$ \\
Sustained political and societal will & $?$
\end{tabular}

Adapted from ref. 95.

viral hepatitis infection (http://www.who.int/csr/disease/hepatitis/ Framework/en/index.html). However, we still have very little information on the number of infected persons, the proportion with cirrhosis or HCC, or the fraction with knowledge of infection. These observations are in striking contrast to the information available for HIV, malaria and other public health priorities. In the United States, although HCV causes more mortality and infects three to four times as many individuals as HIV, appropriations for HCV are less than $2 \%$ of resources for research and for medical care related to HIV ${ }^{94}$. This level of funding is clearly insufficient to drive the type of public health impact that will be possible in the coming years when safer and more efficacious HCV treatments are approved. On a global scale, the projections are even worse, which is of considerable concern given that sustained political and societal will is a key determinant to the success of measures to control infection. Thus, there is no question that HCV will be eliminated increasingly from populations and settings where treatment is provided. Penetrance of testing and treatment into the rest of the world will depend on global commitment.

\section{Conclusion}

HCV infection causes cirrhosis and HCC worldwide; however, for most of the duration of infection, and notably during the interval when treatment is possible, the infection is silent and either unrecognized or ignored by most persons. Improvements in treatment seem to be coming just as the impact of infection begins to rise. However, from a public health perspective, these treatment advances are not likely to have major impact without substantial changes in the methods of control. Efforts to prevent transmission can (and do) work but must be intensified. Treatments can (and do) cure infection but are uncommonly received. In the next decade, there is no doubt that $\mathrm{HCV}$ infection will be eradicated from many individuals. But it is unknown to what extent these exciting new breakthroughs will reach the populations most affected. With sufficient investment, infection could be eradicated from segments of the population and, in principle, the world.

\section{ACKNOWLEDGMENTS}

This work was supported by US National Institutes of Health grant R01 DA013324.

\section{COMPETING FINANCIAL INTERESTS}

The author declares competing financial interests: details are available in the online version of the paper.

Reprints and permissions information is available online at http://www.nature.com/ reprints/index.html.

1. Hoofnagle, J.H. \& Hepatitis, C. The clinical spectrum of disease. Hepatology 26, 15S-20S (1997).

2. Villano, S.A. et al. Persistence of viremia and the importance of long-term followup after acute hepatitis C infection. Hepatology 29, 908-914 (1999).

3. Cox, A.L. et al. Prospective evaluation of community-acquired acute-phase hepatitis C virus infection. Clin. Infect. Dis. 40, 951-958 (2005).

4. Mosley, J.W. et al. Viral and host factors in early hepatitis C virus infection. Hepatology 42, 86-92 (2005). 
5. Lee, M.H. et al. Chronic hepatitis $C$ virus infection increases mortality from hepatic and extrahepatic diseases: a community-based long-term prospective study. J. Infect. Dis. 206, 469-477 (2012).

6. Bruno, S. et al. Hepatitis $C$ virus genotypes and risk of hepatocellular carcinoma in cirrhosis: a prospective study. Hepatology 25, 754-758 (1997).

7. Thomas, D.L. \& Seeff, L.B. Natural history of hepatitis C. Clin. Liver Dis. 9, 383-398 (2005).

8. Goedert, J.J. et al. End-stage liver disease in persons with hemophilia and transfusion- associated infections. Blood 100, 1584-1589 (2002).

9. Thomas, D.L. et al. The natural history of hepatitis $C$ virus infection: host, viral, and environmental factors. J. Am. Med. Assoc. 284, 450-456 (2000).

10. Poynard, T., Bedossa, P. \& Opolon, P. Natural history of liver fibrosis progression in patients with chronic hepatitis C. Lancet 349, 825-832 (1997).

11. Di Bisceglie, A.M. et al. Long-term clinical and histopathological follow-up of chronic posttransfusion hepatitis. Hepatology 14, 969-974 (1991).

12. Farci, P. et al. Profibrogenic chemokines and viral evolution predict rapid progression of hepatitis C to cirrhosis. Proc. Natl. Acad. Sci. USA 109, 14562-14567 (2012).

13. Fierer, D.S. et al. Liver fibrosis during an outbreak of acute hepatitis C virus infection in HIV-infected men: a prospective cohort study. J. Infect. Dis. 198, 683-686 (2008)

14. Agnello, V., Chung, R.T. \& Kaplan, L.M. A role for hepatitis C virus infection in type II cryoglobulinemia. N. Engl. J. Med. 327, 1490-1495 (1992).

15. Mehta, S.H. et al. Prevalence of type 2 diabetes mellitus among persons with hepatitis C virus infection in the United States. Ann. Intern. Med. 133, 592-599 (2000).

16. Spiegel, B.M. et al. Impact of hepatitis $C$ on health related quality of life: a systematic review and quantitative assessment. Hepatology 41, 790-800 (2005).

17. Conrad, S. et al. Living with chronic hepatitis C means 'you just haven't got a normal life any more'. Chronic IIIn. 2, 121-131 (2006).

18. Leigh, J.P. et al. Costs of hepatitis C. Arch. Intern. Med. 161, 2231-2237 (2001).

19. Davis, K.L. et al. Direct economic burden of chronic hepatitis $C$ virus in a United States managed care population. J. Clin. Gastroenterol. 45, e17-e24 (2011).

20. Rein, D.B. et al. The cost-effectiveness of birth-cohort screening for hepatitis C antibody in U.S. primary care settings. Ann. Intern. Med. 156, 263-270 (2012)

21. Liu, S. et al. New protease inhibitors for the treatment of chronic hepatitis C: a cost-effectiveness analysis. Ann. Intern. Med. 156, 279-290 (2012).

22. Mendel, I. et al. Detection and genotyping of the hepatitis C RNA in tear fluid from patients with chronic hepatitis C. J. Med. Virol. 51, 231-233 (1997).

23. Esteban, J.I. et al. High rate of infectivity and liver disease in blood donors with antibodies to hepatitis C virus. Ann. Intern. Med. 115, 443-449 (1991).

24. Seeff, L.B. Hepatitis C from a needlestick injury. Ann. Intern. Med. 115, 411 (1991).

25. Dore, G.J., Kaldor, J.M. \& McCaughan, G.W. Systematic review of role of polymerase chain reaction in defining infectiousness among people infected with hepatitis C virus. Br. Med. J. 315, 333-337 (1997).

26. Thomas, D.L. et al. Perinatal transmission of hepatitis $C$ virus from human immunodeficiency virus type 1-infected mothers. J. Infect. Dis. 177, 1480-1488 (1998).

27. Mast, E.E. et al. Risk factors for perinatal transmission of hepatitis C virus (HCV) and the natural history of HCV infection acquired in infancy. J. Infect. Dis. 192, 1880-1889 (2005).

28. Zanetti, A.R. et al. Mother-to-infant transmission of hepatitis C virus. Lancet $\mathbf{3 4 5}$, 289-291 (1995)

29. Paintsil, E. et al. Survival of hepatitis $C$ virus in syringes: implication for transmission among injection drug users. J. Infect. Dis. 202, 984-990 (2010).

30. Doerrbecker, J. et al. Transmission of hepatitis $C$ virus among people who inject drugs: viral stability and association with drug preparation equipment. J. Infect. Dis. 207, 281-287 (2013).

31. Sartori, M. et al. Transmission of hepatitis C via blood splash into conjunctiva. Scand. J. Infect. Dis. 25, 270-271 (1993).

32. Thomas, D.L. et al. Sexual transmission of hepatitis C virus among patients attending sexually transmitted diseases clinics in Baltimore: an analysis of 309 sex partnerships. J. Infect. Dis. 171, 768-775 (1995)

33. Conry-Cantilena, C. et al. Routes of infection, viremia, and liver disease in blood donors found to have hepatitis C virus infection. N. Engl. J. Med. 334, 1691-1696 (1996).

34. Akahane, Y. et al. Hepatitis $C$ virus infection in spouses of patients with type $C$ chronic liver disease. Ann. Intern. Med. 120, 748-752 (1994).

35. van de Laar, T.J. et al. Sexual transmission of hepatitis $C$ virus in human immunodeficiency virus-negative men who have sex with men: a series of case reports. Sex. Transm. Dis. 38, 102-104 (2011).

36. Terrault, N.A. et al. Sexual transmission of HCV among monogamous heterosexual couples: the HCV partners study. Hepatology 57, 881-889 (2013).

37. Vandelli, C. et al. Lack of evidence of sexual transmission of hepatitis $C$ among monogamous couples: results of a 10-year prospective follow-up study. Am. J. Gastroenterol. 99, 855-859 (2004).
38. Magiorkinis, G. et al. Integrating phylodynamics and epidemiology to estimate transmission diversity in viral epidemics. PLoS Comput. Biol. 9, e1002876 (2013).

39. Williams, I.T. et al. Incidence and transmission patterns of acute hepatitis $C$ in the United States, 1982-2006. Arch. Intern. Med. 171, 242-248 (2011).

40. Hagan, H., Thiede, H. \& Des Jarlais, D.C. Hepatitis C virus infection among injection drug users: survival analysis of time to seroconversion. Epidemiology 15, 543-549 (2004).

41. Schreiber, G.B. et al. The risk of transfusion-transmitted viral infections. The Retrovirus Epidemiology Donor Study. N. Engl. J. Med. 334, 1685-1690 (1996).

42. Thompson, N.D. et al. Nonhospital health care-associated hepatitis B and C virus transmission: United States, 1998-2008. Ann. Intern. Med. 150, 33-39 (2009).

43. Martínez-Bauer, E. et al. Hospital admission is a relevant source of hepatitis C virus acquisition in Spain. J. Hepatol. 48, 20-27 (2008).

44. Hauri, A.M., Armstrong, G.L. \& Hutin, Y.J. The global burden of disease attributable to contaminated injections given in health care settings. Int. J. STD AIDS 15 7-16 (2004).

45. Ko, Y.C. et al. Tattooing as a risk of hepatitis C virus infection. J. Med. Virol. $\mathbf{3 8}$ 288-291 (1992).

46. Mohd Hanafiah, K., Groeger, J., Flaxman, A.D. \& Wiersma, S.T. et al. Global epidemiology of hepatitis $C$ virus infection: new estimates of age-specific antibody to hepatitis C virus seroprevalence. Hepatology 57, 1333-1342 (2013).

47. Frank, C. et al. The role of parenteral antischistosomal therapy in the spread of hepatitis C virus in Egypt. Lancet 355, 887-891 (2000).

48. Strickland, G.T. Liver disease in Egypt: hepatitis C superseded schistosomiasis as a result of iatrogenic and biological factors. Hepatology 43, 915-922 (2006).

49. Guadagnino, V. et al. Hepatitis $C$ virus infection in an endemic area of Southern Italy 14 years later: evidence for a vanishing infection. Dig. Liver Dis. 45 403-407 (2013).

50. Hayashi, J. et al. Transmission of hepatitis $C$ virus by health care workers in a rural area of Japan. Am. J. Gastroenterol. 90, 794-799 (1995).

51. Armstrong, G.L. et al. The prevalence of hepatitis $C$ virus infection in the United States, 1999 through 2002. Ann. Intern. Med. 144, 705-714 (2006).

52. Baillargeon, J. et al. Hepatitis $C$ seroprevalence among newly incarcerated inmates in the Texas correctional system. Public Health 117, 43-48 (2003).

53. Thomas, D.L. et al. Correlates of hepatitis $\mathrm{C}$ virus infections among injection drug users in Baltimore. Medicine 74, 212-220 (1995).

54. Perz, J.F. et al. The contributions of hepatitis $B$ virus and hepatitis $C$ virus infections to cirrhosis and primary liver cancer worldwide. J. Hepatol. 45 529-538 (2006).

55. Lozano, R. et al. Global and regional mortality from 235 causes of death for 20 age groups in 1990 and 2010: a systematic analysis for the Global Burden of Disease Study 2010. Lancet 380, 2095-2128 (2012).

56. Davis, G.L. et al. Aging of hepatitis C virus (HCV)-infected persons in the United States: a multiple cohort model of HCV prevalence and disease progression. Gastroenterology 138, 513-521 (2010).

57. Liao, S.F. et al. Fifteen-year population attributable fractions and causal pies of risk factors for newly developed hepatocellular carcinomas in 11,801 men in Taiwan. PLOS ONE 7, e34779 (2012).

58. El-Serag, H.B. Epidemiology of viral hepatitis and hepatocellular carcinoma. Gastroenterology 142, 1264-1273 (2012).

59. Tanaka, Y. et al. Molecular tracing of the global hepatitis $C$ virus epidemic predicts regional patterns of hepatocellular carcinoma mortality. Gastroenterology 130 703-714 (2006).

60. Schreiber, G.B. et al. The risk of transfusion-transmitted viral infections. The Retrovirus Epidemiology Donor Study. N. Engl. J. Med. 334, 1685-1690 (1996).

61. Mehta, S.H. et al. Changes in blood-borne infection risk among injection drug users. J. Infect. Dis. 203, 587-594 (2011).

62. McHutchison, J.G. et al. Interferon alfa-2b alone or in combination with ribavirin as initial treatment for chronic hepatitis C. N. Engl. J. Med. 339, 1485-1492 (1998).

63. Manns, M.P. et al. Peginterferon alfa-2b plus ribavirin compared with interferon alfa-2b plus ribavirin for initial treatment of chronic hepatitis $\mathrm{C}$ : a randomised trial. Lancet 358, 958-965 (2001).

64. Fried, M.W. et al. Peginterferon alfa-2a plus ribavirin for chronic hepatitis $\mathrm{C}$ virus infection. N. Engl. J. Med. 347, 975-982 (2002).

65. Poordad, F. et al. Boceprevir for intreated chronic HCV genotype 1 infection. N. Engl. J. Med. 364, 1195-1206 (2011).

66. Jacobson, I.M. et al. Telaprevir for previously untreated chronic hepatitis $C$ virus infection. N. Engl. J. Med. 364, 2405-2416 (2011).

67. McHutchison, J.G. et al. Peginterferon alfa-2b or alfa-2a with ribavirin for treatment of hepatitis C infection. N. Engl. J. Med. 361, 580-593 (2009).

68. Shiratori, Y. et al. Histologic improvement of fibrosis in patients with hepatitis $C$ who have sustained response to interferon therapy. Ann. Intern. Med. 132 517-524 (2000)

69. Veldt, B.J. et al. Sustained virologic response and clinical outcomes in patients with chronic hepatitis C and advanced fibrosis. Ann. Intern. Med. 147, 677-684 (2007). 
70. Cardoso, A.C. et al. Impact of peginterferon and ribavirin therapy on hepatocellular carcinoma: incidence and survival in hepatitis $C$ patients with advanced fibrosis. J. Hepatol. 52, 652-657 (2010).

71. Berenguer, J. et al. Sustained virological response to interferon plus ribavirin reduces liver-related complications and mortality in patients coinfected with human immunodeficiency virus and hepatitis C virus. Hepatology 50, 407-413 (2009).

72. van der Meer, A.J. et al. Association between sustained virological response and all-cause mortality among patients with chronic hepatitis $C$ and advanced hepatic fibrosis. J. Am. Med. Assoc. 308, 2584-2593 (2012).

73. Backus, L.I. et al. A sustained virologic response reduces risk of all-cause mortality in patients with hepatitis C. Clin. Gastroenterol. Hepatol. 9, 509-516 (2011).

74. Chou, R. et al. Screening for hepatitis C virus infection in adults: a systematic review for the U.S. Preventive Services Task Force. Ann. Intern. Med. 158, 101-108 (2013).

75. Bernstein, D. et al. Relationship of health-related quality of life to treatment adherence and sustained response in chronic hepatitis C patients. Hepatology 35, 704-708 (2002)

76. John-Baptiste, A.A. et al. Sustained responders have better quality of life and productivity compared with treatment failures long after antiviral therapy for hepatitis C. Am. J. Gastroenterol. 104, 2439-2448 (2009).

77. Liang, J. Nat. Med. 19, aaa-bbb (2013).

78. Patel, P.R et al. Hepatitis C virus infections from a contaminated radiopharmaceutical used in myocardial perfusion studies. J. Am. Med. Assoc. 296, 2005-2011 (2006).

79. Hagan, $\mathrm{H}$. et al. Attribution of hepatitis $\mathrm{C}$ virus seroconversion risk in young injection drug users in 5 US cities. J. Infect. Dis. 201, 378-385 (2010).

80. Hagan, $H$. et al. Reduced risk of hepatitis $B$ and hepatitis $C$ among injection drug users in the Tacoma syringe exchange program. Am. J. Public Health $\mathbf{8 5}$, 1531-1537 (1995).

81. van de Laar, T. et al. Evidence of a large, international network of HCV transmission in HIV-positive men who have sex with men. Gastroenterology 136, 1609-1617 (2009).

82. Grebely, J. et al. Reinfection with hepatitis C virus following sustained virological response in injection drug users. J. Gastroenterol. Hepatol. 25, 1281-1284 (2010).

83. Cottrell, E.B., Chou, R., Wasson, N., Rahman, B. \& Guise, J.-M. Reducing risk for mother-to-infant transmission of hepatitis $C$ virus: a systematic review for the U.S. Preventive Services Task Force. Ann. Intern. Med. 158, 109-113 (2013).

84. Denniston, M.M. et al. Awareness of infection, knowledge of hepatitis C, and medical follow-up among individuals testing positive for hepatitis C: National Health and Nutrition Examination Survey 2001-2008. Hepatology 55, 1652-1661 (2012).

85. Lettmeier, B. et al. Market uptake of new antiviral drugs for the treatment of hepatitis C. J. Hepatol. 49, 528-536 (2008).
86. Volk, M.L. et al. Public health impact of antiviral therapy for hepatitis $C$ in the United States. Hepatology 50, 1750-1755 (2009).

87. Kramer, J.R. et al. Gaps in the achievement of effectiveness of HCV treatment in national VA practice. J. Hepatol. 56, 320-325 (2012).

88. Shivkumar, S. et al. Accuracy of rapid and point-of-care screening tests for hepatitis C: a systematic review and meta-analysis. Ann. Intern. Med. 157, 558-566 (2012).

89. Smith, B.D. et al. Hepatitis C virus testing of persons born during 1945 to 1965 recommendations from the Centers for Disease Control and Prevention. Ann. Intern. Med. 157, 817-822 (2012).

90. Lok, A.S. et al. Preliminary study of two antiviral agents for hepatitis C genotype 1. N. Engl. J. Med. 366, 216-224 (2012).

91. Poordad, F. et al. Exploratory study of oral combination antiviral therapy for hepatitis C. N. Engl. J. Med. 368, 45-53 (2013).

92. Gane, E.J. et al. Nucleotide polymerase inhibitor sofosbuvir plus ribavirin for hepatitis C. N. Engl. J. Med. 368, 34-44 (2013)

93. Sulkowski, M.S. et al. High rate of sustained virologic response with the all-ora combination of daclatasvir plus sofosbuvir, with or without ribavirin in treatmentnaïve patients chronically infected with genotypes 1 , 2, or 3 http://www. natap. org/2012/AASLD/AASLD_06.htm (2012).

94. Edlin, B.R. Perspective: test and treat this silent killer. Nature 474, S18-S19 (2011).

95. Dowdle, W.R. \& Cochi, S.L. The principles and feasibility of disease eradication. Vaccine 29 (suppl. 4), D70-D73 (2011).

96. Rein, D.B.. et al. Forecasting the morbidity and mortality associated with prevalent cases of pre-cirrhotic chronic hepatitis C in the United States. Dig. Liver Dis. 43 66-72 (2011).

97. Wong, J.B. et al. Estimating future hepatitis C morbidity, mortality, and costs in the United States. Am. J. Public Health 90, 1562-1569 (2000).

98. Lehman, E.M. \& Wilson, M.L. Epidemic hepatitis C virus infection in Egypt estimates of past incidence and future morbidity and mortality. J. Viral Hepat. 16, 650-658 (2009).

99. Deuffic, S. et al. Modeling the hepatitis C virus epidemic in France. Hepatology 29, 1596-1601 (1999).

100. Mariano, A. et al. Estimating the incidence, prevalence and clinical burden of hepatitis C over time in Italy. Scand. J. Infect. Dis. 41, 689-699 (2009).

101. Foy, E. et al. Control of antiviral defenses through hepatitis C virus disruption of retinoic acid-inducible gene-I signaling. Proc. Natl. Acad. Sci. USA 102 , 2986-2991 (2005).

102. Barrett, S. et al. The natural course of hepatitis $C$ virus infection after 22 years in a unique homogenous cohort: spontaneous viral clearance and chronic $\mathrm{HCV}$ infection. Gut 49, 423-430 (2001). 